by the microinsertion c.33-34insC, Phe152lle, Gly156Arg then Ile244Thr. Twenty-eight novel variants were detected. None of the 12 new AGXT missense mutations was detected in a population of 50 normal controls.

The whole-gene sequencing method yielded $98 \%$ sensitivity when applied to the 48 probands with liver-biopsy-proven $\mathrm{PH} 1$. Diagnostic sensitivity was $77 \%$ when only the three exons with the highest mutation frequencies were sequenced, still higher than the $62 \%$ sensitivity of a previously published partial-gene screen specific for the Gly170Arg, c.33-34insC and lle244Thr mutations.

These data indicate even greater variety in the AGXT coding region of $\mathrm{PH} 1$ patients than was previously thought. As $A G X T$ is a relatively small gene, the authors believe that whole-gene sequencing is a financially viable technique for molecular diagnosis of $\mathrm{PH} 1$ that could obviate the need for biopsy in many patients.

Original article Monico CG et al. (2007) Comprehensive mutation screening in 55 probands with type 1 primary hyperoxaluria shows feasibility of a gene-based diagnosis. J Am Soc Nephrol 18: 1905-1914

\section{ACE inhibitors do not delay progression of hypodysplastic nephropathy in children}

Angiotensin-converting-enzyme (ACE) inhibitors have been shown to have a renoprotective effect in adults with chronic renal insufficiency (CRI). There is a lack of evidence for similar effects in pediatric patients; nevertheless, ACE inhibitors are commonly used as antiprogression agents in these individuals, despite the very different etiologies, clinical courses and outcomes of CRI in adults and children. Most notably, children with chronic kidney diseases often do not have hypertension or proteinuria.

After analyzing data from the Italian Pediatric Registry of Chronic Renal Failure (ItalKid Project), Ardissino and co-workers have reported no apparent benefits of ACE inhibitors for delaying progression of renal disease in children with hypodysplastic nephropathy, the most common cause of pediatric CRI. In comparison with 123 controls matched by age, gender, diagnosis and baseline creatinine clearance, the rate of progression of CRI (measured as the slope of creatinine clearance over time) was not significantly reduced in 41 children treated with ACE inhibitors. There was, however, a trend towards slower progression in the treatment group (slope $-1.08 \pm 2.08$ vs $-1.80 \pm 4.42 \mathrm{ml} / \mathrm{min} / 1.73 \mathrm{~m}^{2} /$ year; $P=0.31$ ). No significant differences in creatinine clearance slopes were observed when participants were grouped by initial severity of renal impairment, suggesting that selection bias did not influence the results of the analysis.

The authors conclude that use of ACE inhibitors as antiprogression agents should continue to be regarded as experimental in children with hypodysplastic chronic kidney diseases.

Original article Ardissino G et al. (2007) No clear evidence of ACEi efficacy on the progression of chronic kidney disease in children with hypodysplastic nephropathy-report from the ItalKid Project database. Nephrol Dial Transplant [doi: 10.1093/ndt/gfm237]

\section{Polyflux ${ }^{\circledR} 6 \mathrm{H}$ dialyzer: a new option for small children requiring dialysis}

Hemodialysis is the only option in children who require renal replacement therapy but are not candidates for renal transplantation or peritoneal dialysis. During hemodialysis, the extracorporeal circuit should not contain more than $10 \%$ of the patient's blood volume. Until recently, dialyzers meeting this specification for children weighing $<15 \mathrm{~kg}$ were only available from one company in the US (Fresenius Medical Care), but FDA approval has now been obtained for another dialyzer suitable for small children, the Polyflux ${ }^{\circledR} 6 \mathrm{H}$ dialyzer (Gambro AB, Lund, Sweden).

Goldstein et al. evaluated use of this new high-flux dialyzer in six children (mean $\pm \mathrm{SD}$ age $10.3 \pm 3.8$ years, mean weight $24.4 \pm 6.5 \mathrm{~kg}$ ) who had been receiving maintenance hemodialysis with the Fresenius F3 $(n=2), \mathrm{F} 4(n=2)$, or F5 $(n=2)$ dialyzer for at least 2 months. All patients switched to maintenance hemodialysis with the Polyflux ${ }^{\circledR} 6 \mathrm{H}$ dialyzer for 2 consecutive months.

No adverse events occurred during Polyflux ${ }^{\circledR}$ $6 \mathrm{H}$ dialyzer use. Outcome variables (serum sodium, potassium and $\mathrm{CO}_{2}$ levels, post-dialysis weight, total ultrafiltration, single-pool $\mathrm{Kt} / \mathrm{V}$ and estimated equilibrated $K t / V$ did not differ significantly between the pre-evaluation period (with Fresenius dialyzers) and the period of treatment with the Polyflux ${ }^{\circledR} 6 \mathrm{H}$ dialyzer. There 